\title{
OPEN Ambulatory cataract surgery centre without perioperative anaesthesia care: a prospective cohort study
}

\author{
Quentin Duroi ${ }^{1,2}$, Jean-Marie Baudet ${ }^{2}$, Maxime Bigoteau ${ }^{1,2}$, Malek Slim², Tiphanie Pichard ${ }^{2}$, \\ Pierre-Jean Pisella ${ }^{1}$ \& Raoul Kanav Khanna ${ }^{(1,3 \bowtie}$
}

This study aims to evaluate the safety and patient satisfaction of a fast-track procedure for cataract surgery under topical anaesthesia without perioperative anaesthesia care. This is a prospective singlecentre study including all cataract procedures in the Centre Ambulatoire de la Chirurgie de la Cataracte at the Hospital of Bourges between May and August 2018. Procedures were performed under topical anaesthesia without the presence of a nurse anaesthesiologist or anaesthesiologist, the patient had not fasted, and no peripheral venous line was placed. Only heart rate and oxygen saturation were monitored intraoperatively with pulse oximetry. Incidence and nature of intraoperative adverse events and surgical complications were recorded. Patient satisfaction was assessed using the lowa Satisfaction with Anaesthesia Scale (ISAS). In total, 651 cataract surgeries were performed among which $614(94.3 \%)$ were uneventful. Thirty $(4.6 \%)$ intraoperative adverse events and $8(1.2 \%)$ surgical complications were recorded. All surgeries were successfully completed. No medical emergency team intervention or hospital admittance was encountered. The mean ISAS score was 5.7/6, indicating high patient satisfaction. Cataract surgery in an ambulatory cataract surgery centre without perioperative anaesthesia care is a safe procedure with high patient satisfaction for screened patients. Anaesthesia ressources are scarce and may be more beneficial to more complex ophthalmic or non-ophthalmic surgeries.

Cataract surgery is the most commonly performed surgical procedure worldwide. The generalization of phacoemulsification and the progress of less and less invasive surgical techniques, associated with shorter operating times, have gradually led to a reduction in perioperative anaesthetic care. Currently, topical anaesthesia with eye drops is the most widely used method with comparable efficacy to other anaesthesia methods ${ }^{1-4}$. Due to its ease of implementation, topical anaesthesia allows a rapid turnover of patients in the operating room, while avoiding the complications of locoregional anaesthesia ${ }^{2-4}$. Moreover, in recent studies, the need for an anaesthetic procedure during cataract surgery under topical anaesthesia has been shown to be rare ${ }^{5-8}$.

The evaluation report of the French National Authority for Health (HAS) of July 2010 on the "Conditions for performing cataract surgery" paved the way for a simplification of procedures 9 . This report states that a pre-anaesthetic consultation is mandatory in France in the case of general or locoregional anaesthesia, but not mandatory in the case of topical anaesthesia. It also specifies that the use of topical anaesthesia does not require the presence of an anaesthesiologist in the operating room. It is recommended for patient comfort and safety, however, that a referring anaesthesiologist be available when needed.

In France, some teams have begun to offer simplified care for patients undergoing cataract surgery under topical anaesthesia without a systematic preoperative anaesthetic consultation or without an anaesthesiologist present in the operating room ${ }^{10-13}$. This change in practice is in response both to a lack of personnel available to provide conventional anaesthetic management in ophthalmology (preoperative consultation, intraoperative monitoring) and to an increased demand for care given the aging of the population and increased life expectancy.

The Cher area, which capital city is Bourges, is a medical desert (3.2 ophthalmologists per 100,000 inhabitants in 2018, that is to say 10 ophthalmologists; Fig. 1). Since 2015, at the Centre Ambulatoire de la Chirurgie de la Cataracte (CACC) of the Centre Hospitalier Jacques Coeur de Bourges (CHB), eligible patients are operated for cataract surgery on an outpatient basis, under topical anaesthesia, in a dedicated autonomous operating

${ }^{1}$ Department of Ophthalmology, Centre Hospitalier Universitaire Régional de Bretonneau, Bretonneau University Hospital of Tours, 2 Boulevard Tonnellé, 37000 Tours, France. ${ }^{2}$ Department of Ophthalmology, Centre Hospitalier Jacques Coeur, Bourges, France. ${ }^{3}$ INSERM 1253 iBrain «Neurogénomique \& Physiopathologie neuronale», Tours, France. ${ }^{\square}$ email: raoul.khanna@univ-tours.fr 


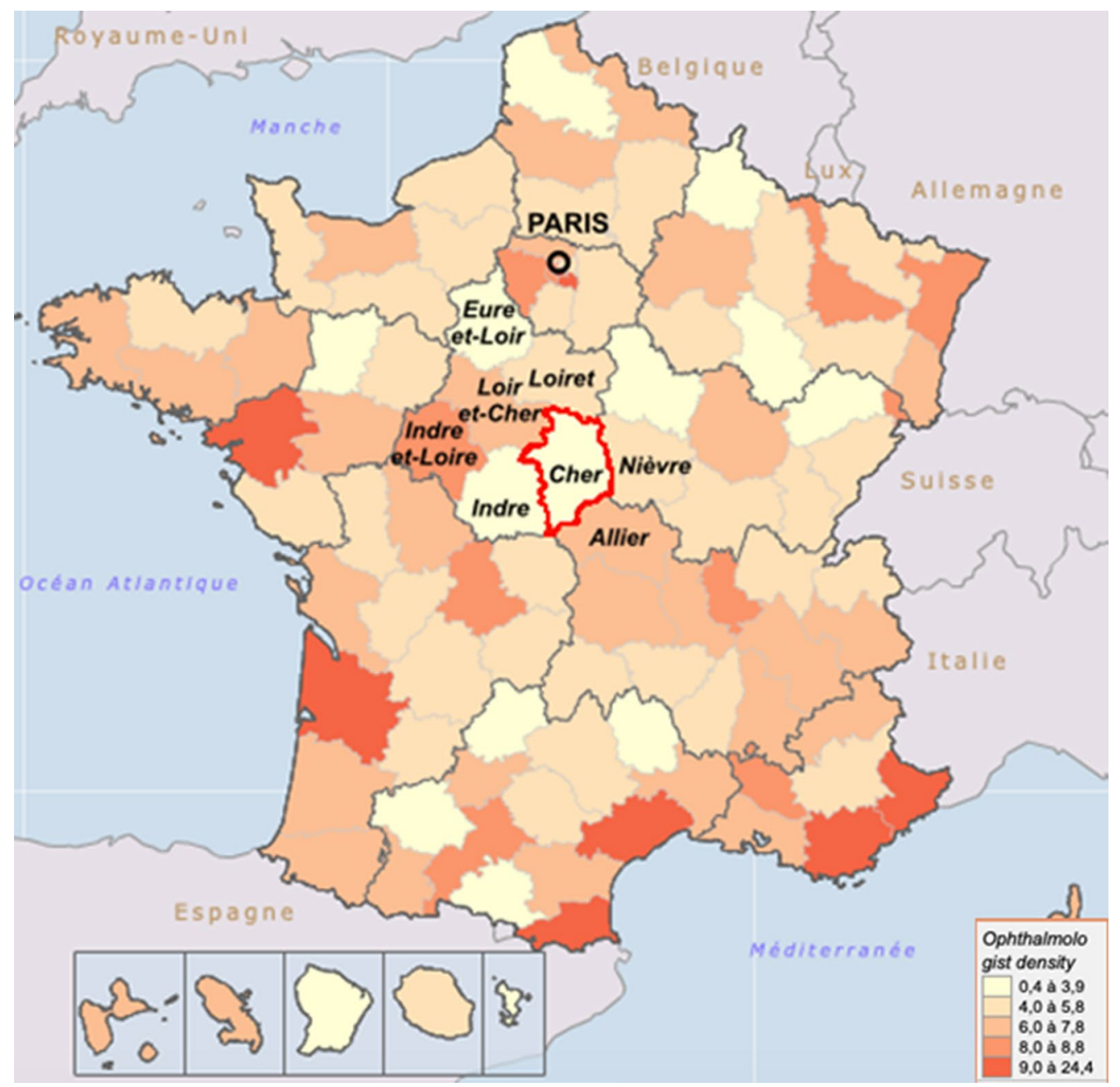

Figure 1. Cartography of ophthalmologist density (according to area) expressed per 100,000 inhabitants (extracted from https://demographie.medecin.fr/mobile.php by MB).

room, without pre-anaesthetic consultation and under the exclusive supervision of the surgical team, without perioperative anaesthetic care. In a previous study, we highlighted the beneficial epidemiological effect of such a structure to improve care in a medical desert ${ }^{14}$.

The main objective of this prospective study was to evaluate the incidence of intraoperative complications and adverse events during cataract surgery in the CACC. The secondary objective was to evaluate patient satisfaction.

\section{Materials and methods}

Study design and population. This prospective single-centre observational study included all patients operated on for cataracts in the CACC at the CHB between May and August 2018. This study was performed using anonymized data and approved by the Ethics Committee of the University Hospital of Tours, France (registration number: 2018034) and the French national commission for information technology and civil liberties (registration number: 2018_062). All subjects provided informed written consent. This study was conducted in accordance with the Jardé law as well as the ethical principles stated in the Helsinki declaration.

Surgical facility. From September 2015, the new ophthalmology department of the CHB, a public general hospital, opened its doors in an independent building. Including on one side the consultations unit and on the other the CACC with an autonomous procedure room dedicated to cataract surgery, allowing most cataract surgeries to be outsourced in the CACC (93.6\% in 2018 according to Bigoteau et al. $\left.2020^{14}\right)$. In agreement with the anaesthesia and surgical teams, patient safety was the responsibility of the CACC team, consisting of a surgeon, a resident and two operating room nurses. In the event of emergency, the Emergency Medical Assistance Service (SAMU) of the CHB could be contacted at any time to respond. The CACC is equipped with an emergency cart, a defibrillator and the necessary drugs for reanimation. CACC staff are regularly trained in first aid techniques and cardiopulmonary reanimation. 


\begin{tabular}{|c|c|c|}
\hline & Location & Procedure \\
\hline \multirow{6}{*}{ Preoperative consultation } & \multirow{6}{*}{ Consultation unit } & Ophthalmic examination \\
\hline & & Medical and surgical history \\
\hline & & Allergies \\
\hline & & Ongoing treatments \\
\hline & & Eligibility for cataract surgery in the CACC \\
\hline & & Information about the chosen care pathway \\
\hline \multirow[b]{2}{*}{ On the eve of the operation } & & Call from the healthcare team \\
\hline & & $\begin{array}{l}\text { Preoperative instructions, hygiene rules, schedules and transport } \\
\text { arrangements }\end{array}$ \\
\hline \multirow{20}{*}{ On the day of surgery } & \multirow{3}{*}{ At home } & The patient is not fasted \\
\hline & & Taking usual medication \\
\hline & & Anxiolytic premedication with alprazolam $0.25 \mathrm{mg}$ \\
\hline & \multirow{2}{*}{ In the CACC } & Admittance \\
\hline & & Check of the compliance with preoperative instructions \\
\hline & Locker room & Storage of personal belongings \\
\hline & \multirow{2}{*}{ Waiting room } & Dilatation (MYDRIATICUM, NÉOSYNÉPHRINE) \\
\hline & & Blood pressure measurement \\
\hline & \multirow{2}{*}{ Transfer airlock } & Seated on a stretcher chair \\
\hline & & No venous route \\
\hline & \multirow{3}{*}{ Operating room } & Topical anesthesia (OXYBUPROCAÏNE, VISTHÉSIA) \\
\hline & & Pulse oxymetry \\
\hline & & Phacoemulsification procedure \\
\hline & \multirow{3}{*}{ Post-operative monitoring room } & Rest \\
\hline & & Pain assessment \\
\hline & & ISAS satisfaction questionnaire \\
\hline & \multirow{2}{*}{ Postoperative consultation } & Slit-lamp examination \\
\hline & & Postoperative recommendations \\
\hline & \multirow{2}{*}{ Conviviality room } & Snack \\
\hline & & Departure \\
\hline On the day after the operation & & Call of the patient by a nurse \\
\hline
\end{tabular}

Table 1. Patient care from the preoperative consultation until the day after the intervention.

Patient track from preoperative to postoperative assessment. The patient track within the CACC is presented in Supplemental file 1 and the main elements of the care pathway are summarized in Table 1.

The preoperative screening criteria excluding treatment within the CACC were as follows: bad cooperation, intractable cough, tremor, respiratory or cardiac insufficiency not permitting a supine position, risk of vasovagal response, ocular disease requiring anaesthetic intervention and impossibility of raising patients. If none of these criteria were observed, the patient was eligible for outpatient surgery under topical anaesthesia at the CACC. In this case, no further medical examination was required and the preoperative anaesthesia consultation was not required. Patients were asked to continue their usual medication and to eat or drink normally on the day of surgery. A prescription for anxiolytic premedication with alprazolam $0.25 \mathrm{mg}$ (XANAX) to be taken the day before and the day of the operation was offered to patients.

In case of systolic blood pressure $\geq 180 \mathrm{mmHg}$ and/or diastolic blood pressure $\geq 110 \mathrm{mmHg}$, the surgeon was warned and could decide to make the patient wait in the waiting room and request a second measurement. If the second measurement remained high, the surgeon could have the nurse administer a single dose of oral antihypertensive drug: nicardipine $20 \mathrm{mg}$ (LOXEN) according to the department's protocol. In the absence of any contraindication after a final measurement, the patient was then admitted to the transfer airlock where they were comfortably seated on the stretcher chair and covered with a warmed sheet. No venous route was placed.

Surgical procedure. In the operating room, the patient was placed in the supine position, with a free-flow of oxygen under the operating drapes and benefited from simple monitoring by pulse oximetry (measurement of heart rate and oxygen saturation). Topical anaesthesia was administered by instillation of anaesthetic eye drops (OXYBUPROCAINE HYDROCHLORIDE $0.4 \%$, Théa $\odot$ ) then by application of a $2 \%$ lidocaine hydrochloride gel (VISTHESIA, Zeiss $\odot$ ) after strict surgical disinfection of the eye with ophthalmic Betadine. The anaesthesia was systematically supplemented at the start of the operation by an intracameral injection of a viscoelastic solution of sodium hyaluronate and $1 \%$ lidocaine hydrochloride (VISTHESIA Intracameral). The surgery was performed by phacoemulsification according to the conventional "Divide and conquer" technique (STELLARIS, Bausch \& Lomb Incorporated( )). Surgery could be performed by the resident accordingly to the senior surgeon's decision. If necessary, verbal and tactile reassurance were provided by the nursing staff in the operating room. 
1. I threw up or felt like throwing up.

2. I would have the same anesthetic again.

3. I itched.

4. I felt relaxed.

5. I felt pain.

6. I felt safe.

7. I was too hot or cold.

8. I was satisfied with the anesthetic care.

9. I felt pain during surgery.

10. I felt good.

11. I hurt.

Figure 2. ISAS, Iowa Satisfaction with Anaesthesia Scale ${ }^{15}$ (copyrighted by Franklin Dexter and the University of Iowa Research Foundation). For items 1, 3, 5, 7, 9, and 11, the scores are calculated as follows: +6 , agree very much; +5 , agree moderately; +4 , agree slightly; +3 , disagree slightly; +2 , disagree moderately; +1 , disagree very much. For items 2, 4, 6, 8, and 10, the scores are calculated in the reverse order from the aforementioned items.

\begin{tabular}{|l|l|l|}
\hline \multicolumn{1}{|l|}{} & & Percentage \\
\hline Patients & 500 & - \\
\hline Age (years) (mean \pm standard deviation) & $74 \pm 8$ & - \\
\hline Women & 303 & 60.6 \\
\hline General comorbidity & 139 & 27.8 \\
\hline 0 & 180 & 36 \\
\hline 1 & 181 & 36.2 \\
\hline$\geq 2$ & 236 & 47.2 \\
\hline High blood pressure & 117 & 23.4 \\
\hline Diabetes mellitus & 130 & 26 \\
\hline Cardiovascular disease & 20 & 4 \\
\hline Thyroid disease & 14 & 2.8 \\
\hline Respiratory disease & 9 & 1.8 \\
\hline Kidney disease & & \\
\hline
\end{tabular}

Table 2. Characteristics of the population.

Data collection. Unusual events encountered by the surgeon were noted on the collection sheet by the surgeon or the nurse, either during or at the end of the procedure. We then collected and classified these events into adverse events or surgical complications. The pain felt during the procedure and before leaving the monitoring room was evaluated by a verbal analogue scale from 0 to 10 (replaced by a simple verbal scale in case of significant visual disturbance). If it was the first eye operated on, the patient indicated whether they would agree to undergo the operation again under the same anaesthetic conditions. A simple verbal satisfaction scale between 0 and 10 and the Iowa Satisfaction with Anaesthesia Scale (ISAS) ${ }^{15,16}$ questionnaire were used. ISAS is a tool for assessing patient satisfaction during cataract surgery which has been validated in French (Fig. 2$)^{17}$. It is a written questionnaire including eleven items dealing with pain, sensations and the anaesthetic experience. With the maximum score being 6 , satisfaction is considered high for a score above $5.4^{18}$. This copyrighted questionnaire is the property of Franklin Dexter and the University of Iowa Research Foundation.

Statistical analyses. Data were collected using Excel software (Microsoft Corporation $\odot$ ). Data collection was double-checked by QD and RKK. Due to the observational nature of our study, no statistical analysis was performed. The quantitative variables were described by the mean \pm standard deviation. Qualitative variables were described by number and percentage.

\section{Results}

In total, 651 surgeries (500 patients; 303 women; mean age: 74 years, range: $43-96)$ were included. Demographic data and comorbidities are described in Table 2 . At least one general comorbidity was identified in $36 \%$ of cases and $36.2 \%$ of subjects had at least two general comorbidities. Anxiolytic premedication by alprazolam $0.25 \mathrm{mg}$ (XANAX) was taken by the patient in $95.7 \%$ of cases. One hundred and fifty-one patients (32\%) underwent successive surgery on both eyes during the study period. This was a second eye surgery for 312 (47.9\%) patients. 


\begin{tabular}{|l|l|l|l|}
\hline & Normal blood pressure & Initially uncontrolled blood pressure & $\begin{array}{l}\text { Initially uncontrolled blood pressure requiring Nicardipine } \\
(\text { LOXEN) }\end{array}$ \\
\hline $\mathrm{N}(\%)$ & $624(95.9)$ & $25(3.8)$ & $2(0.3)$ \\
\hline First measurement & $136 \pm 16$ & $182 \pm 10$ & $201 \pm 12$ \\
\hline Systolic blood pressure $(\mathrm{mmHg})$ & $71 \pm 20$ & $86 \pm 19$ \\
\hline Diastolic blood pressure $(\mathrm{mmHg})$ & $68 \pm 12$ & $152 \pm 15$ & $172 \pm 10$ \\
\hline \multicolumn{5}{|l|}{ Second measurement } & $73 \pm 26$ & $60 \pm 5$ \\
\hline Systolic blood pressure $(\mathrm{mmHg})$ & - & - & \\
\hline Diastolic blood pressure $(\mathrm{mmHg})$ & - &
\end{tabular}

Table 3. Preoperative blood pressure measurements.

\begin{tabular}{|l|l|l|}
\hline & N & Percentage \\
\hline Adverse events & 30 & 4.6 \\
\hline Agitation/anxiety & 16 & 2.5 \\
\hline Bradycardia $(<60)$ & 8 & 1.2 \\
\hline Pain & 4 & 0.6 \\
\hline Oxygen desaturation $(<93 \%)$ & 1 & 0.15 \\
\hline Nausea & 1 & 0.15 \\
\hline Call to the emergency medical assistance service & 0 & - \\
\hline Surgical complications & 8 & 1.2 \\
\hline Iris prolapse & 3 & 0.5 \\
\hline Posterior capsular rupture with anterior vitrectomy & 3 & 0.5 \\
\hline Anterior capsular rupture & 1 & 0.1 \\
\hline Explantation & 1 & 0.1 \\
\hline Posterior lens dislocation & 0 & - \\
\hline No implantation & 0 & - \\
\hline
\end{tabular}

Table 4. Adverse events and surgical complications.

The overall average duration of patient management in the CACC between admission and return home was $125 \pm 32$ min (range: $53-280$ ).

Preoperative blood pressure assessment. The systolic and diastolic blood pressure values at the first measurement, then at the second for uncontrolled blood pressure, are described in Table 3. No patient was discharged for uncontrolled blood pressure.

Surgery. The procedures were performed by 7 surgeons: 3 hospital practitioners and 4 specialist assistants. Surgery was performed by a resident in $7.68 \%$ of cases (50 procedures). The mean duration of the surgeries was $14 \pm 7$ min (range: 5-75). No adverse event or surgical complication was found in 614 cases (94.3\%). The power of the intraocular lens ranged from -3 to +32 . Ninety-three custom-made intraocular lenses $(14.3 \%$, 9 multifocal, 84 toric) were placed.

Adverse events and surgical complications (Table 4). An adverse event occurred for 30 procedures (4.6\%). None of these events led to an interruption of the procedure. No intervention by the SAMU team was necessary. No case of posterior lens dislocation or implantation failure was reported.

Pain, ISAS score and patient satisfaction. The pain experienced during the procedure was evaluated by patients at 0 out of 10 for 586 procedures (90\%). The mean intraoperative pain was $0.2 \pm 0.7$ (range: $0-6$ ) and the mean pain on leaving the monitoring room was $0.1 \pm 0.5$ (range: $0-5$ ). Mean patient satisfaction with the intervention was $9.1 \pm 0.9(2-10)$. The mean ISAS score was $5.66 \pm 0.42$ (range: $3.3-6$ ). Of the 339 individuals operated on for the first eye, 329 indicated that they would like to have the operation for the second eye under the same anaesthetic conditions (97\%).

\section{Discussion}

The procedure described in our study represents a simplified, safe and satisfying care pathway for screened patients eligible for cataract surgery under topical anaesthesia without the following elements: preoperative assessment or blood test, anaesthetic visit, modification of the usual treatments, preoperative fasting, venous line, intravenous sedation. 
Perioperative morbidity and mortality. Although cataract surgery concerns an elderly and often polypathological population, the morbi-moratily rate is very low ${ }^{19}$. Though severe complication have been reported (i.e. cardiac arrest ${ }^{20}$ or severe bradycardia with myocardial infarction ${ }^{21}$ ), these events are extremely rare and the perioperative morbi-mortality is estimated to be between 0.01 and $0.05 \%^{6-8,22}$, rates which are similar to the risk in the general population during daily living activities.

Choice of anaesthesia technique and patient selection. The perioperative anaesthetic care in cataract surgery is still debated and practices vary worldwide ${ }^{23-26}$. Within the CACC, the type of anaesthesia performed is topical anaesthesia. Thus, it is the surgeon's responsibility to decide, for each patient, whether cataract surgery can be performed within the CACC under these conditions. A decision-support screening sheet was designed in partnership with the hospital's anaesthesia team to guide the ophthalmologist. The criteria used are non-binding and the final decision rests with the surgeon, adapting on a case-by-case basis.

The French High Healthy Autority report states that a preoperative anaesthesia consultation is mandatory in the case of general or locoregional anaesthesia whereas it is not always necessary for topical anaesthesia. Performing a systematic preoperative workup before cataract surgery (electrocardiogram and blood test) has shown no benefit in terms of reducing adverse events ${ }^{27}$. Although some patients may be at high risk of complications (e.g. severe diabetes, severe renal dysfunction, severe liver dysfunction), there is good evidence that systematic preoperative blood test prior to cataract surgery does not increase the safety of cataract surgery while increasing $\operatorname{costs}^{27,28}$. Regarding the haemorrhagic complications related to antiplatelet and anticoagulant medications, some studies found a significantly higher incidence of subconjunctival haemorrhage but no significant difference in the incidence of complications nor visual outcomes ${ }^{29,30}$. Thus, these treatments are usually not discontinued before surgery. Preoperative fasting does not appear necessary in case of topical anaesthesia and can even be destabilizing for elderly patients $\mathrm{s}^{31}$. The patients in the current study were instructed to eat normally on the day of surgery and to continue all their usual treatments in order to modify their usual routine as little as possible.

The innovative care pathway set up at the CACC responds to a reasoned use of the available medical resources, while offering patients the appropriate care, adapted and optimized, without unnecessary assessment or therapy. In fact, in a previous study, we showed that this treatment path could be used on a large scale (i.e. $93.6 \%$ of cataract surgeries performed in the CACC in 2018) and was not associated with additional costs compared to the conventional operating room (Supplemental file 2).

Surgery with or without an anaesthesiologist? Several studies have been published on the safety of a protocol without an anaesthesiologist present in the operating room. The measurement of the rate of adverse events and the need for an anaesthesiologist during surgery are the usual endpoints. Bassett et al. compared, in 211 patients, the intraoperative monitoring entrusted to a specially trained nurse versus an anaesthesiologist in terms of patient safety and satisfaction ${ }^{32}$. No serious complications occurred in the two groups and the satisfaction scores were similar. In a study performed by Rocha and Turner, 538 surgeries were realized under oral sedation without an anaesthesiologist present in the operating room but available on demand ${ }^{5}$. In 5 cases $(0.9 \%)$ significant changes in blood pressure required the intervention of the anaesthesiologist. They did not report any surgical complications or unplanned admissions to hospital. In the series of 1002 surgeries reported by Jonas et al., the anaesthesiologist intervened in 29 cases (2.9\%), mainly for hypertensive surge ${ }^{33}$. The largest cohort was published by Zakrzewski et al. Out of 15,440 surgeries performed under the supervision of "registered respiratory therapists", the intervention of an anaesthesiologist was required for 395 procedures $(2.6 \%)^{8}$. Unlike our protocol however, patients received intravenous sedation in most cases.

Only a few French publications relate to protocols without an anaesthesiologist present in the operating room (anaesthetic intervention: Batta et al. 2014a ${ }^{11}: 10 / 124$ (8\%); Bouvet et al. 2015 ${ }^{12}: 20 / 575$ (3.4\%); Jacquens et al. $\left.2020^{34}: 80 / 1,592(5 \%)\right)$. Pepin et al. in their series of 248 cataract surgeries performed under topical anaesthesia, did not find any statistically significant difference in terms of intraoperative pain, surgical complications or postoperative visual acuity between patients operated on with and without an anaesthesiologist present ${ }^{13}$.

Koolwijk et al., in the Netherlands, described a protocol similar to ours on a retrospective series of 6,961 cataract surgeries in an independent outpatient centre, under topical anaesthesia and with minimal intraoperative monitoring (heart rate and oxygen saturation) without the following: pre-anaesthetic consultation, anaesthesiologist in the operating room, venous line ${ }^{35}$. They reported 3 emergency team interventions $(0.04 \%)$ for postoperative vasovagal collapse and no hospitalization was necessary. Murray et al., in a large retrospective series of 6,661 surgeries, performed without anaesthetic supervision over an 8-year period, described only one serious adverse event ${ }^{22}$. They concluded that the majority of cataract surgery procedures under local anaesthesia could be performed safely without the presence of an anaesthesiologist.

Adverse events and intraoperative complications. Intraoperative pain is the most frequently reported adverse event in the literature with surgery under local anaesthesia ${ }^{5,11,16,18}$. The pain experienced is also a determining factor in patient satisfaction ${ }^{18}$. Rocha et al. reported minimal or moderate intraoperative pain in $13 \%$ of cases ${ }^{5}$, which is higher than our results $(0.6 \%)$. The systematic addition of intracamerular lidocaine to numb the iris has been shown to be effective in achieving additional analgesia and may partly explain this good result $^{3}$. The mean intraoperative pain evaluated in our study was less than $1 / 10$, indicating minimal pain, acceptable to the patient, and $90 \%$ of subjects found the intervention painless.

The most common intercurrent event found in our study was agitation/anxiety which was successfully managed by providing tactile and verbal reassurance. In fact, no surgical complications were noted in patients who exhibited anxiety. Some teams $\mathrm{s}^{5-8}$ use intravenous sedation in case of patient agitation, but systematic venous line and monitoring by an anaesthesiologist would then legally be required in France in that case, which was not 
possible in our protocol. Placing a venous line can be a painful and stressful experience for patients, especially in the elderly whose veins can be fragile ${ }^{36}$. Additionally, Katz et al. demonstrated, in their study of nearly 20,000 procedures, a significant increase in the frequency of intraoperative adverse events with intravenous sedation ${ }^{6}$. The complication rate was $0.13 \%$ with pure topical anaesthesia, $0.78 \%$ with locoregional anaesthesia, and it increased to $1.2 \%$ when intravenous sedation was combined with topical anaesthesia or locoregional anaesthesia. On the other hand, several publications have shown that low doses of oral anxiolytic can be used without risk to effectively reduce pre- and intraoperative anxiety ${ }^{5,35,37}$.

A surge in hypertension during surgery is one of the most common reason for intraoperative anaesthesiologist intervention, occurring in 0.5 to $5 \%^{5-8,10,11,33}$. High blood pressure was found in almost half of our patients (47\%). Yet, almost all of our patients had satisfactory blood pressure from the start (96\%) which can be explained by the adherence to the usual medication intake and the absence of preoperative fasting.

Posterior capsular rupture with or without vitreous loss can cause major operating difficulties, compromise visual outcome and is considered as the main parameter to assess safety ${ }^{25,26}$. The incidence of posterior capsular rupture ranges between 0.5 and 5.1\% $\%^{5,11,25,26,35,38}$ and has been shown to be correlated with the surgeon's experience $^{26}$. In our study, experienced hospital practitioners, specialist assistants and residents performed the procedures and the rate of posterior capsular rupture was low $(0.5 \%)$.

Patient satisfaction. The measurement of patient satisfaction is an interesting tool for evaluating the quality of care and, by taking into account the patient experience, improving practices ${ }^{39}$. Fung et al., $2005^{18}$, and Batta et al., $2014^{11}$, reported respectively an ISAS score of 5.6 and 5.46 which is consistent with our findings of 5.66. The factors influencing patient satisfaction are known to be pain, anxiety, surgeon's skills, but also duration of care $^{4,18}$. The patient track in the CACC is intended to be rapid and optimized to reduce waiting times, a potential source of stress and annoyance, to the lowest level. The average duration of overall management between admission and return home was $2 \mathrm{~h}$ which was certainly appreciated by the patients.

Demographic and economic aspects. The CHB is located in the heart of the Cher area, a medical desert ranked $96^{\text {th }}$ among the 109 French areas in terms of ophthalmologist density. The population of the Cher area is older than the national average and the demand for cataract surgery is high. In a previous study, we demonstrated that the CACC had a positive local epidemiological impact by increasing attractiveness and decreasing leakage of patients to other territories without additionnal costs compared to the conventional operating room (Supplemental file 2$)^{14}$.

Limitations. The current study has certain limitations. Due to the absence of blood pressure measurement during the operation, any hypertensive surges could not be detected, although this is an adverse event frequently reported in the literature, which may require the intervention of the anaesthesiologist. Further comparative studies are needed to address the role of intraoperative blood pressure measurement as it is not yet known if it can improve safety and outcomes. Yet, the incidence of adverse events remained low and similar to the findings reported by Koolwijk et al. ${ }^{35}$. Visual disturbances were experienced by patients immediately after surgery, and they often had difficulty filling out the questionnaires on their own. The nurse in the monitoring room routinely offered to help patients, which may have influenced their responses. However, this bias was also present in other studies $^{11,16,18}$. This study was performed in an ambulatory surgery centre located within a public general hospital and thus further evaluation is needed for private practice.

This study shows that cataract surgery can be performed safely with a high degree of patient satisfaction in an outpatient setting under topical anaesthesia, without perioperative anaesthetic care or venous line, under minimal intraoperative monitoring and without an anaesthesiologist immediately available. Preoperative screening is essential, but it appears that a large majority of patients can benefit from this type of procedure. Due to the low rate of adverse events and the absence of serious medical complications during cataract surgery under topical anaesthesia, monitoring by an anaesthesia team does not appear essential. An emergency medical team must, however, be reachable and available to respond rapidly if necessary. The protocol used at the CACC offers safe and satisfactory care for the patient, without increasing costs. Surgical activity, however, depends on the organizational specificities of each healthcare establishment and on the motivation of the surgical and anaesthesia teams.

\section{Data availability}

The datasets generated during and/or analysed during the current study are available from the corresponding author on reasonable request.

Received: 16 December 2020; Accepted: 22 March 2021

Published online: 15 April 2021

\section{References}

1. Alhassan, M. B., Kyari, F., \& Ejere, H. O. Peribulbar versus retrobulbar anaesthesia for cataract surgery. Cochrane Database of Systematic Reviews CD004083 (2008)

2. Davison, M., Padroni, S., Bunce, C., \& Rüschen, H. Sub-Tenon's anaesthesia versus topical anaesthesia for cataract surgery. Cochrane Database of Systematic Reviews CD006291 (2007)

3. Ezra, D. G., \& Allan, B. D. Topical anaesthesia alone versus topical anaesthesia with intracameral lidocaine for phacoemulsification. Cochrane Database of Systematic Reviews CD005276 (2007)

4. Zhao, L.-Q., Zhu, H., Zhao, P.-Q., Wu, Q.-R. \& Hu, Y.-Q. Topical anesthesia versus regional anesthesia for cataract surgery: A meta-analysis of randomized controlled trials. Ophthalmology 119, 659-667 (2012). 
5. Rocha, G. \& Turner, C. Safety of cataract surgery under topical anesthesia with oral sedation without anesthetic monitoring. Can. J. Ophthalmol. J. Can. Ophtalmol. 42, 288-294 (2007).

6. Katz, J. et al. Adverse intraoperative medical events and their association with anesthesia management strategies in cataract surgery. Ophthalmology 108, 1721-1726 (2001).

7. Zakrzewski, P. A., Friel, T., Fox, G. \& Braga-Mele, R. Monitored anesthesia care provided by registered respiratory care practitioners during cataract surgery: a report of 1957 cases. Ophthalmology 112, 272-277 (2005).

8. Zakrzewski, P. A., Banashkevich, A. V., Friel, T. \& Braga-Mele, R. Monitored anesthesia care by registered respiratory therapists during cataract surgery: an update. Ophthalmology 117, 897-902 (2010).

9. Conditions de réalisation de la chirurgie de la cataracte: environnement technique—rapport dévaluation. Haute Autorité de Santé. https://www.has-sante.fr/jcms/c_992026/fr/conditions-de-realisation-de-la-chirurgie-de-la-cataracte-environnement-techniquerapport-d-evaluation.

10. de Beketch, C. et al. Protocole simplifié de prise en charge anesthésique chez les patients opérés de cataracte sous topique: étude rétrospective sur un an. J. Fr. Ophtalmol. 36, 50-54 (2013).

11. Batta, B., Fuchs-Buder, T., Tréchot, F. \& Angioi, K. Étude de satisfaction et de sécurité d'un protocole d’anesthésie simplifié pour la chirurgie de cataracte sous anesthésie topique. J. Fr. Ophtalmol. 37, 548-556 (2014).

12. Bouvet, L. et al. Évaluation du recours à l'anesthésiste-réanimateur lors de la chirurgie de la cataracte réalisée sous anesthésie topique. J. Fr. Ophtalmol. 38, 316-321 (2015).

13. Pepin, M., Rineau, E., Caignard, A., Leruez, S. \& Gohier, P. Lanesthésie topique sans recours à un médecin anesthésiste dans la chirurgie de la cataracte ambulatoire de l'adulte: évaluation des critères de sélection en consultation, à propos de 248 cas. J. Fr. Ophtalmol. 41, 441-446 (2018).

14. Bigoteau, M. et al. Ambulatory surgery centers: possible solution to improve cataract healthcare in medical deserts. J. Cataract Refract. Surg. 47, 352-357 (2020).

15. Dexter, F., Aker, J. \& Wright, W. A. Development of a measure of patient satisfaction with monitored anesthesia care: The Iowa Satisfaction with Anesthesia Scale. Anesthesiology 87, 865-873 (1997).

16. Fung, D., Cohen, M., Stewart, S. \& Davies, A. Can the Iowa satisfaction with Anesthesia Scale be used to measure patient satisfaction with cataract care under topical local anesthesia and monitored sedation at a community hospital?. Anesth. Analg. 100, 1637 (2005).

17. Falempin, A.-S. et al. Transcultural validation of a French version of the Iowa Satisfaction with Anesthesia Scale (ISAS-F). Can. J. Anesth. Can. Anesth. 67, 541-549 (2020).

18. Fung, D., Cohen, M. M., Stewart, S. \& Davies, A. What determines patient satisfaction with cataract care under topical local anesthesia and monitored sedation in a community hospital setting?. Anesth. Analg. 100, 1644-1650 (2005).

19. Greenberg, P. B. et al. Predictors of mortality within 90 days of cataract surgery. Ophthalmology 117, 1894.el-1899.e1 (2010).

20. Hadden, P. W. \& Scott, R. C. Cardiac arrest during phacoemulsification using topical anesthesia in an unsedated patient. J. Cataract Refract. Surg. 32, 369 (2006).

21. Hampl, K. F., Marsch, S. C., Schneider, M. \& Flammer, J. Vasovagal heart block following cataract surgery under local anesthesia. Ophthalmic Surg. 24, 422-424 (1993).

22. Murray, P., Adams, K., Haddad, P., Murray, N. \& O'Rourke, M. The routine requirement for anaesthetists in local anaesthetic cataract surgery. Clin. Exp. Ophthalmol. 35, 195-196 (2007).

23. Nørregaard, J. C. International variation in anesthesia care during cataract surgery: Results from the international cataract surgery outcomes study. Arch. Ophthalmol. 115, 1304 (1997).

24. Reeves, S. W. et al. A decision analysis of anesthesia management for cataract surgery. Am. J. Ophthalmol. 132, 528-536 (2001).

25. Jaycock, P. et al. The Cataract National Dataset electronic multi-centre audit of 55,567 operations: Updating benchmark standards of care in the United Kingdom and internationally. Eye Lond. Engl. 23, 38-49 (2009).

26. Johnston, R. L., Taylor, H., Smith, R. \& Sparrow, J. M. The Cataract National Dataset electronic multi-centre audit of 55,567 operations: Variation in posterior capsule rupture rates between surgeons. Eye Lond. Engl. 24, 888-893 (2010).

27. Keay, L., Lindsley, K., Tielsch, J., Katz, J. \& Schein, O. Routine preoperative medical testing for cataract surgery. Cochrane Database Syst. Rev. 1, CD007293 (2019).

28. Alboim, C. et al. The impact of preoperative evaluation on perioperative events in patients undergoing cataract surgery: A cohort study. Eye Lond. Engl. 30, 1614-1622 (2016).

29. Benzimra, J. D. et al. The Cataract National Dataset electronic multicentre audit of 55,567 operations: Antiplatelet and anticoagulant medications. Eye Lond. Engl. 23, 10-16 (2009).

30. Kobayashi, H. Evaluation of the need to discontinue antiplatelet and anticoagulant medications before cataract surgery. J. Cataract Refract. Surg. 36, 1115-1119 (2010).

31. Sanmugasunderam, S. \& Khalfan, A. Is fasting required before cataract surgery? A retrospective review. Can. J. Ophthalmol. J. Can. Ophtalmol. 44, 655-656 (2009).

32. Bassett, K. et al. Nurse anaesthetic care during cataract surgery: A comparative quality assurance study. Can. J. Ophthalmol. J. Can. Ophtalmol. 42, 689-694 (2007).

33. Jonas, J. B., Pakdaman, B., Sauder, G. \& Bender, H.-J. Is intraoperative monitoring necessary in cataract surgery under topical anesthesia?. J. Cataract Refract. Surg. 30, 2645-2646 (2004).

34. Jacquens, A. et al. Safe short circuit in cataract surgery: Incidence and risk factors for intraoperative medical action. Anaesth. Crit. Care Pain Med. 39, 611-616 (2020).

35. Koolwijk, J. et al. Outpatient cataract surgery: Incident and procedural risk analysis do not support current clinical ophthalmology guidelines. Ophthalmology 122, 281-287 (2015).

36. Walsh, G. Difficult peripheral venous access: Recognizing and managing the patient at risk. J. Assoc. Vasc. Access 13, 198-203 (2008).

37. Chen, M., Hill, G. M., Patrianakos, T. D., Ku, E. S. \& Chen, M. L. Oral diazepam versus intravenous midazolam for conscious sedation during cataract surgery performed using topical anesthesia. J. Cataract Refract. Surg. 41, 415-421 (2015).

38. Lebuisson, D. A. \& Jolivet, M.-C. L’anesthésie topique en chirurgie de la cataracte ambulatoire de ladulte sans anesthésiste présent. J. Fr. Ophtalmol. 28, 59-67 (2005).

39. Chanthong, P., Abrishami, A., Wong, J., Herrera, F. \& Chung, F. Systematic review of questionnaires measuring patient satisfaction in ambulatory anesthesia. Anesthesiology 110, 1061-1067 (2009).

\section{Acknowledgements}

We thank the University Hospital of Tours for providing the services of a native English speaker who reviewed the whole manuscript and the tables (A.D.T. International - L'Agence de Traduction). 


\section{Author contributions}

Q.D., J.M.B., M.B., M.S., T.P., P.J.P., R.K.K. were involved in drafting the article or revising it critically for important intellectual content, and all authors approved the final version to be published. R.K.K. had full access to all of the data in the study and takes responsibility for the integrity and the accuracy of the data.

\section{Competing interests}

The authors declare no competing interests.

\section{Additional information}

Supplementary Information The online version contains supplementary material available at https://doi.org/ 10.1038/s41598-021-87926-0.

Correspondence and requests for materials should be addressed to R.K.K.

Reprints and permissions information is available at www.nature.com/reprints.

Publisher's note Springer Nature remains neutral with regard to jurisdictional claims in published maps and institutional affiliations.

Open Access This article is licensed under a Creative Commons Attribution 4.0 International License, which permits use, sharing, adaptation, distribution and reproduction in any medium or format, as long as you give appropriate credit to the original author(s) and the source, provide a link to the Creative Commons licence, and indicate if changes were made. The images or other third party material in this article are included in the article's Creative Commons licence, unless indicated otherwise in a credit line to the material. If material is not included in the article's Creative Commons licence and your intended use is not permitted by statutory regulation or exceeds the permitted use, you will need to obtain permission directly from the copyright holder. To view a copy of this licence, visit http://creativecommons.org/licenses/by/4.0/.

(C) The Author(s) 2021, corrected publication 2021 